\title{
Role of miR-181a in the process of apoptosis of multiple malignant tumors: A literature review
}

\author{
Xialu Feng ${ }^{A-C, E, F}$, Chen Zhang ${ }^{A-C, F}$, Yan Yang ${ }^{B-D, F}$, Deren Hou ${ }^{C, D, F}$, Anding Zhu ${ }^{C, D, F}$ \\ Department of Neurology, Third Xiangya Hospital, Central South University, Changsha, China \\ A - research concept and design; $B$ - collection and/or assembly of data; $C$ - data analysis and interpretation; \\ $D$ - writing the article; $E$ - critical revision of the article; $F$ - final approval of the article
}

Address for correspondence

Chen Zhang

E-mail:2016chenzhang@sina.com

\section{Funding sources}

The study was supported by the New Xiangya Talent Projects of the Third Xiangya Hospital of Central South University (JY201624).

Conflict of interest

None declared

Received on June 18, 2016

Reviewed on September 1, 2016

Accepted on November 8, 2016

\begin{abstract}
It has been recognized that miR-181a expression is dysregulated and intimately associated with clinical prognosis in a variety of human cancers. However, the direct role of miR-181a in tumor progression has been elusive. Moreover, mounting evidence has demonstrated that cellular apoptosis, a physiological process of programmed cell death, is disrupted in various categories of human malignancies. Multiple apoptosisrelated genes have been proven to act as the target genes of miR-181a. In this study, we hypothesize that miR-181a probably plays a potential role in modulating the procession and apoptosis of cancer cells. We performed a literature review and elucidated how miR-181a modulated cellular apoptosis, especially the malignant neoplasm cells. We also unraveled the potential role of miR-181a in the diagnosis, treatment and clinical prognosis of multiple human malignancies - miR-181a plays a pivotal role in the development, treatment and prognosis of patients suffering from malignant tumors. It also participates in the development of cancer partially by modulating cellular apoptosis.
\end{abstract}

Key words: miR-181a, apoptosis, Bcl-2 family, P53, PRKCD

DOI

$10.17219 /$ acem/66842

\section{Copyright}

Copyright by Author(s)

This is an article distributed under the terms of the

Creative Commons Attribution Non-Commercial License

(http://creativecommons.org/licenses/by-nc-nd/4.0/) 


\section{Introduction}

MicroRNAs (miRNAs) are defined as a class of highlyconserved, small non-coding RNA (22 nucleotides on average), which function to modulate gene expression at the posttranscriptional level by binding to the 3'-untranslated region (3'-UTR) of target genes. Previous investigations have demonstrated that the regulatory role of miRNAs is involved in multiple cellular processes, including cellular metabolism, proliferation, differentiation, development, and apoptosis. Aberrant expression of miRNAs contributes to the incidence and progression of malignant tumors, whereas the precise role of miRNAs in the development of malignant neoplasms remains elusive. ${ }^{1}$ As one of multiple conserved miRNAs among vertebrates, miR-181a has been confirmed to be differentially expressed in a variety of diseases, especially various cancers, and plays a critical role in the occurrence and development of malignant neoplasms.

Apoptosis is a physiological process of programmed cell death that is essential for normal tissue development and homeostasis, through which damaged, unattached, mutant, and aged cells are eliminated. Mounting evidence has demonstrated that miR-181a is capable of modulating cellular apoptosis by targeting several apoptosis-related genes, probably related to the underlying mechanism of the role of miR-181a involved in tumor progression. Aberrations in the signaling pathway can lead to degenerative and autoimmune disorders, and even cancer. ${ }^{2}$ This paper reviews the mechanism underlying the role of miR181-a involved in cellular apoptosis and investigates the correlation between miR-181a and varying human malignancies.

\section{Apoptosis}

Since apoptosis was first described in 1842 by Carl Vogt, accumulated studies have been performed to deepen the understanding of cellular apoptosis. As one of 2 major types of cell death, apoptosis is a highly-regulated process with specific and well-described morphological changes, and is critical for many physiological processes, including cell development, proliferation, differentiation, immune regulation, and eliminating defective and harmful cells. Dysfunction of apoptosis is central to multiple pathological states. For instance, enhanced apoptosis has been described in neurodegenerative diseases, e.g., acquired immunodeficiency syndrome (AIDS), transplant rejection and heart failure, whereas diminished apoptosis has been observed in autoimmune diseases, such as viral infections and even cancers. ${ }^{3-7}$

Currently, cancer is one of the major causes of a poor quality of life and human death. Common anticancer therapies, such as chemo- and radiotherapy, mainly induce the apoptosis of cancer cells by administration of cytotoxic agents. In 2014, Gong et al. demonstrated that Nexrutine ${ }^{\circledR}$ treatment could inhibit the growth of pancreatic cancer cells through induction of apoptosis. ${ }^{8}$ Targeting components of the apoptotic pathway has been regarded as a therapeutic approach for cancers, because aberrant apoptosis is central to the growth of malignant tumors and the resistance to anticancer therapies. Failures in normal apoptotic pathways contribute to carcinogenesis by creating a permissive environment for genetic instability and accumulation of gene mutations. In turn, tumor cells employ a variety of molecular mechanisms to suppress apoptosis, thus establishing a tumor/cancer-promoting loop. ${ }^{9}$

With deepening the understanding of the relationship between apoptosis and cancer, researchers have recently found that aberrant expression of miRNAs in tumor cells results in the dysfunction of cellular apoptosis. For instance, miR-106a overexpression significantly aggravated cellular apoptosis induced by cisplatin in ovarian cancer A2780 cells through downregulating the expression of antiapoptotic protein Mcl-1. ${ }^{10}$ Additionally, Ribeiro et al. summarized the function of human miRNAs in carcinogenesis and target genes. ${ }^{11}$ The let-7 family acts as a tumor suppressor by targeting c-Myc. It has been proven that the miR-15-16 cluster is an oncogene by interacting with $\mathrm{Bcl}-2$. Interestingly, miR-125b plays a dual role, serving as an oncogene by regulating p 53 and Bak-1 expression, whereas acting as a tumor suppressor during interaction with Bcl2, Mcl-1, Bcl-w, etc. All these miRNA targets, including Bcl-2, Bcl-w, Bak-1, and Mcl-1, are capable of encoding the proteins associated with cellular apoptosis. Consequently, apoptosis dysfunction is implicated in the progression of malignant tumors. Especially in cancer cells, miRNAs are potentially capable of regulating the process of cellular apoptosis.

\section{miR-181a and cancer}

As a member of the miR-181 family, miRNA-181a has been intensively studied; miRNA-181a is located on the chromosome 1 (37.p5) and miR-181a2 is situated on the chromosome 9 (37.p5). Similar to other miRNAs, miR-181a plays a pivotal role in many cellular processes, such as cell fate determination and cellular invasion..$^{12}$ It has been discovered to be abnormally expressed in solid malignant tumors and hematological malignancies. A significant downregulation of miR-181a level has been detected in squamous lung cell carcinoma, oral squamous cell carcinoma, salivary adenoid cystic carcinoma, acute myeloid leukemia, and non-small cell lung cancer, whereas evident overexpression of miR-181a level has been found in MCF-7 breast cancers, multiple myeloma, pancreatic and gastric cancer, and hepatocellular carcinoma. ${ }^{13-22}$ All these investigations have demonstrated that aberrant expression of miR-181a contributes to the development and metastasis of malignant tumors. In patients diagnosed with 
colorectal cancer, miR-181a overexpression promotes CRC cell growth, invasion and liver metastasis by targeting the 3'-UTR of tumor suppressor gene WIF1. ${ }^{23}$ Additionally, upregulated expression of miRNA-181a can induce carcinogenesis by targeting E2F5 in hepatocellular carcinoma evolved from chronic hepatitis B. ${ }^{24}$

Additionally, it has been revealed that high expression of miR-181a is associated with short recurrence time and poor outcome in patients with epithelial ovarian cancer. ${ }^{25}$ In a meta-analysis investigation, Lin et al. suggested that low expression of miR-181a/b is significantly associated with poor survival in patients with hematological malignancies. ${ }^{26}$

Decades of research have indicated that miR-181a plays a critical role in tumorigenesis and cancer progression, whereas the mechanism underling this function remains to be unraveled. Some scholars have indicated that, as a regulator of tumor phenotype, miR-181a is involved in tumorigenesis and downstream malignant processes through the ability to modulate the expression of critical genes and signaling networks which are mainly involved with regulating cell apoptosis. ${ }^{25}$ Hence, the influence on apoptosis is believed to be a vital mechanism for miR-181a to participate in tumor progression and development. In this review, the mechanisms underlying the role of miR-181a in cancer cellular apoptosis were retrospectively analyzed, and the correlation between miR-181a and the diagnosis, treatment and prognosis of malignant tumors was also briefly discussed.

\section{miR-181a modulates apoptosis by targeting apoptosis-related genes}

To mediate cellular apoptosis, 2 signaling pathways have been accepted: extrinsic and intrinsic. ${ }^{27}$ The extrinsic pathway of apoptosis is mediated by ligands activating death receptors, such as APO2L/TRAIL, FasL(Fasl/ /APOI/CD95), TNF(TNFR1), etc. The intrinsic pathway of apoptosis is initiated in the mitochondria, and tightly regulated by the balance between pro-apoptotic (e.g., Bcl-1, Bax, Bim, and P53) and anti-apoptotic genes (e.g., Bcl-1 and Mcl-1). Caspases, such as cysteine aspartic acid specific protease, serve as the central regulatory proteins in both extrinsic and intrinsic signaling pathways. Aberrant expression of regulatory genes results in deregulation of these pathways and subsequently leads to the incidence of multiple diseases. Recent research has verified that miR-181a could modulate cell apoptosis by targeting the apoptosisrelated genes, including Bcl-2 family, P53, ATM, PRKCD, $P B X 3$, and RALA (Table 1).

\section{Bcl-2 gene family}

The Bcl-2 gene family plays a pivotal role in the regulation of apoptosis via the mitochondrial pathway. The proteins of the Bcl-2 family are encoded, and consist
Table 1. Target genes and effect of miR-181a upon cellular apoptosis

\begin{tabular}{|c|c|c|c|}
\hline Target & Relationship & Tissues & Activity \\
\hline \multirow{6}{*}{$\mathrm{BCl}-2$} & \multirow{5}{*}{ negative } & breast cancer cell ${ }^{30}$ & \multirow{5}{*}{ enhance } \\
\hline & & malignant glioma cell ${ }^{32}$ & \\
\hline & & CLL cell ${ }^{34}$ & \\
\hline & & AML cell ${ }^{35}$ & \\
\hline & & prostate cancer cell ${ }^{37}$ & \\
\hline & positive & osteosarcoma cell ${ }^{31}$ & inhibit \\
\hline $\mathrm{Mcl}-1$ & negative & CLL cell ${ }^{40}$ & enhance \\
\hline Bax & positive & NSCLC cell ${ }^{41}$ & enhance \\
\hline Bim & negative & lymphoma cell ${ }^{42}$ & inhibit \\
\hline \multirow{2}{*}{ P53 } & \multirow{2}{*}{ positive } & myeloma cell ${ }^{19}$ & \multirow{2}{*}{ enhance } \\
\hline & & CLL cell ${ }^{40}$ & \\
\hline ATM & negative & gastric cancer cell ${ }^{44}$ & inhibit \\
\hline PRKCD & negative & $\begin{array}{l}\text { squamous cervical } \\
\text { carcinoma cell }^{48,49}\end{array}$ & inhibit \\
\hline $\mathrm{PBX} 3$ & negative & $\begin{array}{c}\text { acute myelogenous } \\
\text { leukemia }\end{array}$ & enhance \\
\hline RalA & negative & $\begin{array}{l}\text { chronic myelogenous } \\
\text { leukemia }\end{array}$ & enhance \\
\hline
\end{tabular}

of approx. 20 pro- and anti-apoptotic proteins falling into 3 groups. Anti-apoptotic multidomain members, such as Bcl-2, Mcl-1 and Bcl-w, contain BH1-4 domains, whereas pro-apoptotic multidomain proteins, including the Bax subfamily, contain BH1-3 domains. The BH3-only group, such as Bim, is proven to be pro-apoptotic. These proteins contribute to cellular apoptosis by regulating mitochondrial permeability, fission and fusion. Recent investigations have demonstrated that these proteins play a role in cellular homeostasis with respect to metabolism, calcium signaling, endoplasmic reticulum function, and autophagy. ${ }^{28,29}$

\section{B-cell lymphoma 2}

B-cell lymphoma 2 (Bcl-2), encoded by the $B c l 2$ gene in humans, is the founding member of the Bcl-2 family. It has been recognized that miR-181a can suppress $\mathrm{Bcl}-2$ expression by targeting the 3 '-UTR of the $\mathrm{Bcl}-2$ gene. ${ }^{30}$ In spite of the negative relationship, western blotting has shown that the upregulation of $\mathrm{Bcl}-2$ in MG63 cells is associated with the overexpression of miR-181a. ${ }^{31}$ In conclusion, miR-181a contributes to inducin gand suppressing apoptosis via interaction with the $\mathrm{Bcl}$-2 gene.

Chen et al. suggested that miR181a sensitizes human malignant glioma cells to radiation by targeting Bcl-2. ${ }^{32}$ MTT assay detected that cell growth rate was significantly reduced in miR-181a overexpressed cells after 18.8 Gy irradiation. Furthermore, they proved that the upregulation of miR-181a (exogenous miR-181a expression) resulted in a decrease in the expression of $\mathrm{Bcl}-2$ protein. Khanna et al.proposed that overexpression of miR-34a, miR-30e and miR-181a could 
increase the rate of apoptosis, accompanied by a decline in Bcl-2 expression in miRNA-transfected mouse models. ${ }^{33}$ $\mathrm{Li}$ et al. constructed a luciferase reporter vector and identified a target site of miR-181a in the BCL-2 3' UTR. ${ }^{34} \mathrm{~K} 562$ cells transfected with an miR-181a inhibitor had significantly higher survival compared to normal K562 cells, and K562/A02 cells transfected with a miR-181a mimic had a significantly lower survival than non-transfected K562/A02 cells, implying that miR181a can decrease the survival rate of the CLL cells treated by daunorubicin via targeting Bcl-2, regardless of whether the CLL cells are sensitive or resistant to daunorubicin. Bcl-2 was confirmed as a direct miR-181a target by immunoblot analysis and reporter gene assays. Bai et al. found that the Bcl-2 3'UTR contains a highlyconserved 8 mer site complementary to the seed region of the miR-181a. ${ }^{35}$ Moreover, they demonstrated that miR-181a can intensify caspase-dependent cell death through Bcl-2 in AML cells. Ouyang et al. established a mouse model and found that, compared to control cells, elevated expression of miR-181a reduced cell survival by $31 \%$, whereas knockdown of endogenous miR-181a levels increased survival by $27 \%{ }^{36}$ Moreover, the downregulated levels of miR-181a are associated with reduced cell death and oxidative stress, and preserved mitochondrial function in astrocytes via upregulating Bcl-2 protein level. Zhai et al. observed that the expression levels of miR-181a in PC-3 prostatecancer cells were upregulated after bufalin treatment, indicating that inhibition of miR-181a activity could attenuate bufalin-induced apoptosis in PC-3 cells. ${ }^{37}$ Furthermore, miR-181a inhibitor can reverse the reduction of bufalin-induced Bcl-2, whereas miR-181a transfection was shown to downregulate the expression of Bcl-2 significantly, suggesting that induced miR-181a mediates downstream bufalin-induced apoptosis by repressing Bcl-2 protein in PC-3 cells. Moon et al. confirmed that N2a cells subjected to serum deprivation and oxidative stress yielded less cell death when the expression of miR-181a was downregulated, whereas it aggravated cell death at the upregulation of miR-181a levels. ${ }^{38}$

\section{Myeloid cell leukemia-1}

Myeloid cell leukemia-1 (Mcl-1) is the second member of the Bcl-2 family, which directly interacts with the $\mathrm{BH} 3$ alpha-helical domain of pro-apoptotic proteins, such as Bax, Bak, Bad, and Bim, and inhibits their functions. ${ }^{39}$ Ouyang et al. and Zhu et al. revealed that Bcl-1 is not only a direct target of miR-181a, but also miR-181a could enhance the cell apoptosis via negative interaction with $\mathrm{Mcl}-1 .{ }^{36,40}$

\section{Bax}

As an apoptosis regulator, Bax, also known as Bcl-2like protein 4 , is a human protein encoded by the $B a x$ gene. $\mathrm{Bax}$ is a member of the $\mathrm{Bcl}-2$ gene family and promotes apoptosis by binding to and antagonizing the Bcl-2
protein.Galluzzi et al. suggested that miR-181a is capable of sensitizing NSCLC A549 cells to the lethal action of CDDP, carboplatin and oxaliplatin by stimulating Bax oligomerization, and the activation of pro-apoptotic caspases. ${ }^{41}$ Khanna et al. observed that the miR-34a, miR-30e and miR-181a not only modulate Bcl-2 expression, but downregulate the expression of pro-apoptosis genes, such as Bax, and cleavage of caspases, and then gain neuronal survival in the brain of calorie-restricted mice. ${ }^{33}$ In brief, the positive relationship between miR-181a and Bax validates that miR-181a plays a pro-apoptotic role in both human and mouse cells.

\section{Bim}

Bim, as a member of the $\mathrm{BH} 3$-only family and $\mathrm{Bcl}$-a protein family, contains only 1 single $\mathrm{BH}$-domain. Bim plays a key role in promoting apoptosis. Lwin et al. demonstrated that adhesion of mantle cell lymphoma and other non-Hodgkin lymphoma cells to follicular dendritic cells reduced cell apoptosis and was associated with downregulated levels of Bim. ${ }^{42}$ Moreover, cell adhesion is capable of upregulating the expression of miR-181a; miR-181a overexpression decreased, whereas miR-181a inhibition increased Bim levels by directly targeting Bim. These results imply that miR-181a acts as a negative effector of the Bim-apoptosis signaling pathway.

\section{Tumor protein p53}

Tumor protein $\mathrm{p} 53(\mathrm{p} 53)$ is a transcription factor that activates or represses the expression of multiple genes. Numerous studies have established that p53 promotes apoptosis by transcriptionally activating or repressing the expression of a panel of pro- and anti-apoptotic proteins. Additionally, activation of p53-dependent apoptosis leads to mitochondrial apoptotic changes via both intrinsic and extrinsic pathways, triggering cell death notably by the release of cytochrome $\mathrm{c}$ and activation of caspase cascade. ${ }^{43}$

Pichiorri et al. demonstrated that the miR-181a targets p300-CBP-associated factor and through p53 indirectly controls p53 activity in myeloma, and functions as a positive regulator of $\mathrm{p} 53 .{ }^{1 \times 9}$ Furthermore, Zhu et al. revealed that chronic lymphocytic leukemia cells transfected with miR-181a from p53 wild-type patients led to a significant increase in apoptosis, compared to miRNA controls. ${ }^{40}$ However, enforced expression of miR-181a exerted no effect on B-CLL cells from p53-attenuated patients, implying that miR-181a can enhance cellular apoptosis by targeting p53.

\section{Ataxia telangiectasia mutated}

Ataxia telangiectasia mutated (ATM) is a serine/threonineprotein kinase that is recruited and activated by DNA double-strand breaks. ATM phosphorylates several 
key proteins that initiate activation of the DNA damage checkpoint, leading to cell cycle arrest, DNA repair or apoptosis. Several of these targets, including p53, CHK2 and $\mathrm{H} 2 \mathrm{AX}$, are tumor suppressors. Zhang et al. demonstrated that ATM is a direct target of miR-181a, miR-181a mimics transfection downregulating the expression of ATM at both mRNA and protein levels. ${ }^{44}$ Additionally, compared to negative control and blank groups, transfection of miR-181a inhibitor is capable of inhibiting proliferation, invasion and migration, while promoting the apoptosis of SGC7901 cells. This data collectively indicates that overexpression of miR-181a promotes the proliferation, while suppressing the apoptosis of gastric cancer cells through directly targeting ATM.

\section{Protein kinase $\mathrm{C}$ delta type}

Protein kinase $C$ delta type (PKC- $\delta$ ), an enzyme encoded by the $P R K C D$ gene, acts as a substrate for caspase-3. A series of studies have shown that $P R K C D$ activity is required for apoptosis induced by DNA damaging agents, such as cisplatin, etoposide, cytosine arabinoside, mitomycin $\mathrm{C}$, and doxorubicin. The regulating effect of PRKCD upon cellular apoptosis is highly complex. PKC- $\delta$ participates in oxLDL-induced endoplasmic reticulum stress-dependent apoptotic signaling through the IRE1 $\alpha /$ JNK pathway. ${ }^{45}$ Moreover, PKC- $\delta$ plays a crucial role in the propagation of TNF $\alpha$-induced endoplasmic reticulum stress-mediated JNK activation and CHOP/GADD53 induction. ${ }^{46}$ Recent research has revealed that the expression of $P R K C D$ is modulated by miR-181a. Bergman et al. have demonstrated that the PRKCD gene is a direct target of miR-181a in a rat model of multiple sclerosis. ${ }^{47} \mathrm{Ke}$ et al. and Chen et al. found that miR-181a could inhibit the irradiation- and cisplatininduced apoptosis of human squamous cervical carcinoma cells via downregulating the expression levels of $P R K C D{ }^{48,49}$

\section{PBX3}

$P B X 3$, a HOXA cofactor gene, can encode pre-B-cell leukemia transcription factor 3 , which is capable of regulating the transcription of downstream targets by forming stable heterocomplexes with HOX and MEIS proteins. In human leukemic cells carrying $M L L$ rearrangements, ectopic expression of miR-495 significantly inhibits cell viability and increases cell apoptosis via directly targeting the $P B X 3$ gene, implying that $P B X 3$ could regulate cellular apoptosis, but the underling mechanism remains elusive. ${ }^{50} \mathrm{Li}$ et al. demonstrated that miR-181a possesses the same function as miR-495. ${ }^{51}$ Upregulated expression of miR-181a significantly promotes cellular apoptosis, inhibits the viability and proliferation of leukemic cells, and delays leukemogenesis by downregulating the endogenous expression of $P B X 3$ at both the RNA and protein levels.
The effect of miR-181a upon the growth and apoptosis of leukemic cells depends on the PBX3 signaling pathway.

\section{RalA}

RalA, as an important effector of Ras, is proven to be involved in tumorigenesis and cancer invasion, and overactivated in multiple human cancers, such as malignant peripheral nerve sheath tumor, non-small cell lung cancer and chronic myeloid leukemia. ${ }^{52}$ Male et al. demonstrated that the proliferation and invasiveness of A549 cells were reduced upon silencing RalA, whereas apoptosis and necrosis were enhanced in such conditions in non-small cell lung cancer cell lines. ${ }^{53} \mathrm{Zhu}$ et al. investigated that siRNA RalA, used to reduce RalA protein level in K562 and KCL-22 cells, effectively inhibited cell viability by significantly increasing caspase 3 activity, and CML cells transfected with siRNA RalA acquired typical features of apoptosis, including nuclear pyknosis, fragmentation and apoptotic body at 48 -h post-transfection. ${ }^{54}$ This evidence validated that downregulation of RalA can induce cell apoptosis. Fei et al. demonstrated that miR-181a could downregulate the expression of RalA. ${ }^{55}$ The dual-luciferase reporter and western blot assays confirmed that RalA contains a miR-181a binding site at its 3'-UTR and is directly regulated by miR-181a. Additionally, immunoblot and RT-PCR revealed that overexpression of miR-181a significantly downregulates the expression level of RalA mRNA, subsequently suppresses cell growth, and eventually induces G2-phase arrest and apoptosis in leukemia K562 cells.

\section{Programmed cell death 4}

The programmed cell death 4 (PDCD4) gene functions to encode a protein localized within the nucleus in proliferating cells. The product of PDCD4 is thought to play a role in apoptosis, but the specific role has not been determined. Previous investigations have indicated that PDCD4 protein was downregulated in HCC tissues, and Huh7 cells transfected with PDCD4 resulted in upregulated expression of cytosolic cytochrome complex (cyt c) and mitochondrial Bax accompanied by downregulated levels of mitochondrial cyt $c$ and cytosolic Bax. Furthermore, a slight reduction of procaspase-8, and a significant reduction of procaspase- 9 and procaspase- 3 were observed after PDCD4 transfection. These results indicate that PDCD4 might induce apoptosis via mitochondria events and caspase cascade. Additionally, the PDCD 4 gene is originally identified as a tumor-related gene in humans and acts as a tumor suppressor in mouse epidermal carcinoma cells, prompting that the suppressing effect of PDCD4 upon malignant tumors could be regulated by miR-21 and miR-106a. ${ }^{56-58}$ Daisuke et al. demonstrated that PDCD4 is a target gene of miR-181a, and the increased miR-181a levels were 
significantly associated with shortened disease-free survival and overall survival of breast cancer patients, whereas low expression of PDCD4 was significantly correlated with poorer disease-free survival. ${ }^{59}$ However, no statistical significance was observed between $P D C D 4$ gene expression and accumulation of miR-181a. The regulating effect upon cellular apoptosis is complex, and deeper understanding of the relationship between miR-181a and PDCD 4 and other apoptosis-related genes is urgently required.

\section{Conclusions}

The mechanism underlying the role of miRNAs involved in the pathogenesis of malignant neoplasm remains an emphasis. Malignant neoplasm represents a broad group of diseases involving unregulated cell growth, implying that abnormal cellular apoptosis plays a pivotal role in the development of malignancies.

In this paper, we conducted a literature review to elucidate how miR-181a modulates cellular apoptosis. Specifically, miR-181a modulates apoptosis by targeting the apoptosis-related genes. The enhancing/inhibiting apoptosis balance is probably explained by the direct interaction between miR-181a and alternative apoptosis-related genes. It has been recognized that miR-181a is capable of enhancing apoptosis of cells, particularly in malignant tumor cells, by targeting p53, Bax, Bcl-2, PBX3, and RalA, while suppressing apoptosis by interacting with PRKCD, ATM, Bim, and Bcl-2.

Depending on the different functions involved in the apoptotic process and aberrant expression in cancer cells, miR-181a could dually act as oncogene and tumor suppressors, as illustrated in Table 2. Despite the fact that miR-181a is

Table 2. Relationship between miR-181a and development and apoptosis of malignant tumors

\begin{tabular}{|c|c|c|c|}
\hline $\begin{array}{c}\text { Expression } \\
\text { of } \\
\text { miR-181a }\end{array}$ & Tissues & $\begin{array}{l}\text { Regulation } \\
\text { on } \\
\text { apoptosis } \\
\text { process }\end{array}$ & Activity \\
\hline \multirow{6}{*}{ Reduced } & malignant glioma cell 32 & \multirow{6}{*}{ enhance } & \multirow{6}{*}{ oncogene } \\
\hline & CLL cell $\|^{34,40}$ & & \\
\hline & AML cell ${ }^{16,52,51}$ & & \\
\hline & CML cell ${ }^{55}$ & & \\
\hline & prostate cancer cell ${ }^{37,60}$ & & \\
\hline & NSCLC cell15,41 & & \\
\hline \multirow{6}{*}{ Increased } & osteosarcoma cell ${ }^{31}$ & \multirow{4}{*}{ inhibit } & \multirow{4}{*}{ oncogene } \\
\hline & lymphoma cell|22 & & \\
\hline & gastric cancer cell|21,44 & & \\
\hline & $\begin{array}{l}\text { squamous cervical } \\
\text { carcinoma cell|48,49 }\end{array}$ & & \\
\hline & breast cancer cell 8,30 & \multirow{2}{*}{ enhance } & \multirow{2}{*}{$\begin{array}{c}\text { tumor } \\
\text { suppressor }\end{array}$} \\
\hline & myeloma cell19 & & \\
\hline
\end{tabular}

involved in cancer development, overexpression of miR-181a sensitizes cancer cells to drugs and radiation via targeting apoptosis-related genes. ${ }^{29,31,36,42}$ Additionally, miR-181a contributes to the bufalin-induced apoptosis and cisplatin-induced apoptosis of cancer cells. ${ }^{35,38}$ Based on the role of miR-181a in chemotherapy or radiotherapy, miR-181a might be a potential target for the treatment of varying cancers. The predictive role miR-181a plays in epithelial ovarian cancer and hematological malignancies verifies that miR-181a levels could be used as a potential prognostic biomarker predicting the clinical prognosis of cancer patients.

Taken together, miR-181a plays a pivotal role in the development, treatment and prognosis of patients suffering from malignant tumors. It participates in the development of cancer partially by modulating cellular apoptosis. Nevertheless, the exact role of miR-181a in different malignancies remains to be elucidated. If miR-181a could serve as a clinical parameter, prognostic biomarkers in different types of cancer should be subsequently validated by clinical investigations.

\section{References}

1. Baer C, Claus R, Plass C. Genome-wide epigenetic regulation of miRNAs in cancer. Cancer Res. 2013;73(2):473-477.

2. Wong RS. Apoptosis in cancer: From pathogenesis to treatment. JExp Clin Cancer Res. 2011;30:87.

3. Cheng XL, Li MK. Effect of topiramate on apoptosis-related protein expression of hippocampus in model rats with Alzheimers disease. Eur Rev Med Pharmacol Sci. 2014;18(6):761-768.

4. Joshi A, Lee RT, Mohl J, et al. Genetic signatures of HIV-1 envelopemediated bystander apoptosis. J Biol Chem. 2014;289(5):2497-2514. doi:10.1074/jbc.M113.514018

5. Wang Y, Wu J, Jiang B, et al. Relationship between ischemia/reperfusion injury and acute rejection of allogeneic liver transplant in rats. Transplant Proc. 2014;46(1):50-55.

6. Zeng $Z$, Shen $\mathrm{L}$, $\mathrm{Li}$ X, et al.Disruption of histamine $\mathrm{H} 2$ receptor slows heart failure progression through reducing myocardial apoptosis and fibrosis. Clin Sci (Lond). 2014;127(7):435-448.

7. Ouyang L, Shi Z, Zhao S, et al. Programmed cell death pathways in cancer: A review of apoptosis, autophagy and programmed necrosis. Cell Prolif. 2012;45(6):487-498.

8. Gong J, Xie J, Bedolla R, et al. Combined targeting of STAT3/NF-KB/ COX-2/EP4 for effective management of pancreatic cancer. Clin Cancer Res. 2014;20(5):1259-1273.

9. Sun Y, Peng ZL. Programmed cell death and cancer. Postgrad Med J. 2009;85(1001):134-140.

10. Yao YM, Shi HR, Ji M, Chen CH. MiR-106a targets Mcl-1 to suppress cisplatin resistance of ovarian cancer A2780 cells. J Huazhong Univ Sci Technolog Med Sci. 2013;33(4):567-572.

11. Ribeiro J, Sousa H. MicroRNAs as biomarkers of cervical cancer development: A literature review on miR-125b and miR-34a. Mol Biol Rep. 2014;41(3):1525-1531.

12. Neel JC, Lebrun JJ. Activin and TGF $\beta$ regulate expression of the microRNA-181 family to promote cell migration and invasion in breast cancer cells. Cell Signal. 2013;25(7):1556-1566.

13. Gao W, Shen H, Liu L, Shu Y. MiR-21 overexpression in human primary squamous cell lung carcinoma is associated with poor patient prognosis. J Cancer Res Clin Oncol. 2011;137:557-566.

14. Shin KH, Bae SD, Hong HS, Kim RH, Kang MK, Park NH. MiR-181a shows tumor suppressive effect against oral squamous cell carcinoma cells by downregulating K-ras. Biochem Biophys Res Commun. 2011;404:896-902.

15. Gao W, Yu Y, Cao H, Shen H, Li X, Pan S. Deregulated expression of miR-21, miR-141 and miR-181a in non small cell lung cancer is relat- 
ed to clinicopathologic characteristics or patient prognosis. Biomed Pharmacother. 2010;64:399-408.

16. Wu Y, Li XF, Yang JH, Liao XY, Chen YZ. microRNAs expression profile in acute promyelocytic leukemia cell differentiation induced by all-trans retinoic acid and arsenic trioxide. Zhonghua Xue Ye Xue Za Zhi. 2012;33(7):546-551.

17. He Q, Zhou X, Li S, et al. MicroRNA-181a suppresses salivary adenoid cystic carcinoma metastasis by targeting MAPK-Snai2 pathway. Biochim Biophys Acta. 2013;1830(11):5258-5266.

18. Jiao X, Zhao L, Ma M, et al. MiR-181a enhances drug sensitivity in mitoxantone-resistant breast cancer cells by targeting breast cancer resistance protein (BCRP/ABCG2). Breast Cancer Res Treat. 2013;139(3): 717-730.

19. Pichiorri F, Suh SS, Ladetto $M$, et al. MicroRNAs regulate critical genes associated with multiple myeloma pathogenesis. Proc Natl Acad Sci US A. 2008;105:12885-12890.

20. Liu J, Xu D, Wang Q, Zheng D, Jiang X, Xu L. LPS induced miR-181a promotes pancreatic cancer cell migration via targeting PTEN and MAP2K4. Dig Dis Sci. 2014;59(7):1452-1460.

21. Zhang X, Nie Y, Du Y, Cao J, Shen B, Li Y. MicroRNA-181a promotes gastric cancer by negatively regulating tumor suppressor KLF6. Tumour Biol. 2012;33(5):1589-1597.

22. Brockhausen J, Tay SS, Grzelak CA. miR-181a mediates TGF- $\beta$-induced hepatocyte EMT and is dysregulated in cirrhosis and hepatocellular cancer. Liver Int. 2014;35(1):240-253. doi:10.1111/liv.12517

23. Ji D, Chen Z, Li M, et al. MicroRNA-181a promotes tumor growth and liver metastasis in colorectal cancer by targeting the tumor suppressor WIF-1. Mol Cancer. 2014;13(1):86.

24. Zou C, Li Y, Cao Y, et al. Up-regulated MicroRNA-181a induces carcinogenesis in hepatitis $B$ virus-related hepatocellular carcinoma by targeting E2F5. BMC Cancer. 2014;14:97. doi:10.1186/1471-2407-14-97

25. Parikh A, Lee $C$, Joseph $P$, et al. microRNA-181a has a critical role in ovarian cancer progression through the regulation of the epithelial-mesenchymal transition. Nat Commun. 2014;5:2977. doi:10.1038/ ncomms3977

26. Lin S, Pan L, Guo S, et al. Prognostic role of microRNA-181a/b in hematological malignancies. A meta-analysis. PLoS One. 2013;8(3):e59532. doi:10.1371/journal.pone.0059532

27. Khan KH. Cancer therapeutics: Targeting the apoptosis pathway. Crit Rev Oncol Hematol. 2014;90(3):200-219.

28. Danial NN, Gimenez-Cassina A, Tondera D. Homeostatic functions of BCL-2 proteins beyond apoptosis. Adv Exp Med Biol. 2010;687:1-32.

29. Rolland SG, Conradt B. New role of the BCL2 family of proteins in the regulation of mitochondrial dynamics. Curr Opin Cell Biol. 2010;22:852-858.

30. Zhu Y, Wu J, Li S, et al. The function role of miR-181a in chemosensitivity to adriamycin by targeting $\mathrm{Bcl}-2$ in low-invasive breast cancer cells. Cell Physiol Biochem. 2013;32(5):1225-1237.

31. Jianwei Z, Fan L, Xiancheng L, Enzhong B, Shuai L, Can L. MicroRNA 181a improves proliferation and invasion, suppresses apoptosis of osteosarcoma cell. Tumour Biol. 2013;34(6):3331-3337.

32. Chen G, Zhu W, Shi D, et al. MicroRNA-181a sensitizes human malignant glioma U87MG cells to radiation by targeting Bcl-2. Oncol Rep. 2010;23(4):997-1003.

33. Khanna A, Muthusamy S, Liang R, Sarojini H, Wang E. Gain of survival signaling by down-regulation of three key miRNAs in brain of calorie-restricted mice. Aging (Albany NY). 2011;3(3):223-236.

34. Li H, Hui L, Xu W. MiR-181a sensitizes a multidrug-resistant leukemia cell line K562/A02 to daunorubicin by targeting BCL-2. Acta Biochim Biophys Sin (Shanghai). 2012;44(3):269-277.

35. Bai H, Cao Z, Deng C, Zhou L, Wang C. MiR-181a sensitizes resistant leukaemia HL-60/Ara-C cells to Ara-C by inducing apoptosis. J Cancer Res Clin Oncol. 2012;138(4):595-602.

36. Ouyang YB, Lu Y, Yue S, Giffard RG. miR-181 targets multiple Bcl-2 family members and influences apoptosis and mitochondrial function in astrocytes. Mitochondrion. 2012;12(2):213-219.

37. Zhai XF, Fang FF, Liu Q, Meng YB, Guo YY, Chen Z. MiR-181a contributes to bufalin-induced apoptosis in PC-3 prostate cancer cells. BMC Complement Altern Med. 2013;13:325.

38. Moon JM, Xu L, Giffard, RG. Inhibition of microRNA-181 reduces forebrain ischemia-induced neuronal loss. J Cereb Blood Flow Metab. 2013;33(12):1976-1982.
39. Kazi A, Sun J, Doi K, et al. The BH3 alpha-helical mimic BH3-M6 disrupts $B c l-X(L), B c l-2$, and $M C L-1$ protein-protein interactions with Bax, Bak, Bad, or Bim and induces apoptosis in a Bax-and Bim-dependent manner. J Biol Chem. 2011;286:9382-9392.

40. Zhu DX, Zhu W, Fang C, et al. miR-181a/b significantly enhances drug sensitivity in chronic lymphocytic leukemia cells via targeting multiple anti-apoptosis genes. Carcinogenesis. 2012;33(7):1294-1301.

41. Galluzzi L, Morselli E, Vitale I, et al. miR-181a and miR-630 regulate cisplatin-induced cancer cell death. Cancer Res. 2010;70(5):1793-1803.

42. Lwin T, Lin J, Choi YS, et al. Follicular dendritic cell-dependent drug resistance of non-Hodgkin lymphoma involves cell adhesion-mediated Bim down-regulation through induction of microRNA-181a. Blood. 2010;116(24):5228-5236.

43. Wang DB, Kinoshita C, Kinoshita Y, Morrison RS. p53 and mitochondrial function in neurons. Biochim Biophys Acta. 2014;1842(8): 1186-1197.

44. Zhang $X$, Nie $Y$, Li X, et al. MicroRNA-181a functions as an oncomir in gastric cancer by targeting the tumour suppressor gene ATM. Pathol Oncol Res. 2014;20(2):381-389. doi:10.1007/s12253-0139707-0

45. Larroque-Cardoso P, Swiader A, Ingueneau C, et al. Role of protein kinase $C \delta$ in ER stress and apoptosis induced by oxidized LDL in human vascular smooth muscle cells. Cell Death Dis. 2013;28:4:e520.

46. Greene MW, Ruhoff MS, Burrington CM, Garofalo RS, Orena SJ. TNF alpha activation of PKC delta, mediated by NFkappaB and ER stress, cross-talks with the insulin signaling cascade. Cell Signal. 2010;22:274-284

47. Bergman $P$, James $T$, Kular $L$, et al. Next-generation sequencing identifies microRNAs that associate with pathogenic autoimmune neuroinflammation in rats. J Immunol. 2013;190(8):4066-4075. doi:10.4049/ jimmunol.1200728

48. Ke G, Liang L, Yang JM, et al. MiR-181a confers resistance of cervical cancer to radiation therapy through targeting the pro-apoptotic PRKCD gene. Oncogene. 2013;32(25):3019-3027. doi:10.1038/ onc.2012.323

49. Chen Y, Ke G, Han D, Liang S, Yang G, Wu X. MicroRNA-181a enhances the chemoresistance of human cervical squamous cell carcinoma to cisplatin by targeting PRKCD. Exp Cell Res. 2014;320(1):12-20.

50. Jiang $X$, Huang $\mathrm{H}$, Li Z, et al. MiR-495 is a tumor-suppressor microRNA down-regulated in MLL-rearranged leukemia. Proc Natl Acad Sci USA. 2012;109(47):19397-19402. doi:10.1073/pnas.1217519109

51. Li Z, Huang H, Li Y, et al. Up-regulation of a HOXA-PBX3 homeoboxgene signature following down-regulation of miR-181 is associated with adverse prognosis in patients with cytogenetically abnormal AML. Blood. 2012;119(10):2314-2324. doi:10.1182/blood-201110-386235

52. Borrego-Diaz E, Terai K, Lialyte K, et al. Overactivation of Ras signaling pathway in CD133+ MPNST cells. J Neurooncol. 2012;108(3):423434. doi:10.1007/s11060-012-0852-1

53. Male $H$, Patel V, Jacob MA, et al. Inhibition of RalA signaling pathway in treatment of non-small cell lung cancer. Lung Cancer. 2012;77(2):252-259.

54. Zhu X, Li Y, Luo X, Fei J. Inhibition of small GTPase RalA regulates growth and arsenic-induced apoptosis in chronic myeloid leukemia (CML) cells. Cell Signal. 2012;24(6):1134-1140. doi:10.1016/j.cellsig.2012.01.016

55. Fei J, Li Y, Zhu X, Luo X. miR-181a post-transcriptionally downregulates oncogenic RalA and contributes to growth inhibition and apoptosis in chronic myelogenous leukemia (CML). PLoS One. 2012;7(3):e32834

56. Yang GD, Huang TJ, Peng LX, et al. Epstein-Barr virus_encoded LMP1 upregulates microRNA-21 to promote the resistance of nasopharyngeal carcinoma cells to cisplatin-induced apoptosis by suppressing PDCD4 and Fas-L. PLoS One. 2013;8(10):e78355.

57. Ren W, Wang X, Gao L, et al. miR-21 modulates chemosensitivity of tongue squamous cell carcinoma cells to cisplatin by targeting PDCD4. Mol Cell Biochem. 2014;390(1-2):253-262. doi:10.1007/s11010014-1976-8

58. Li H, Xu H, Shen H, Li H. microRNA-106a modulates cisplatin sensitivity by targeting PDCD4 in human ovarian cancer cells. Oncol Lett. 2014;7(1):183-188. 
59. Ota D, Mimori K, Yokobori T, et al. Identification of recurrence-related microRNAs in the bone marrow of breast cancer patients. Int J Oncol. 2011:38:955-962.

60. Su SF, Chang YW, Andreu-Vieyra C, et al. miR-30d, miR-181a and miR-199a-5p cooperatively suppress the endoplasmic reticulum chaperone and signaling regulator GRP78 in cancer. Oncogene. 2013;32(39):4694-4701. 